\title{
Case of Breast Cancer Discovered by Repeated Hematomas after Blunt Trauma to the Breast
}

\author{
Jingjing Wang* \\ The First Affiliated Hospital of Anhui Medical University, China
}

*Corresponding author: Jingjing Wang, The First Affiliated Hospital of Anhui Medical University, 218 Jixi Road, Shushan District, Hefei City, Anhui Province, China

To Cite This Article: Jingjing Wang. Case of Breast Cancer Discovered by Repeated Hematomas after Blunt Trauma to the Breast. Am J Biomed Sci \& Res. 2021 - 12(6). AJBSR.MS.ID.001808. DOI: 10.34297/AJBSR.2021.12.001808.

Received: 制 April 29, 2021; Published: 眥 May 17, 2021

\begin{abstract}
Background: Breast hematoma is a manifestation of blunt force injury to the breast. Hematomas without obvious clinical complications were treated conservatively and most of them were self-healing. If there is no history of tumor and no need for emergency treatment, the possibility of tumor is often ignored by clinicians due to the history of trauma. The repeated hematoma in this case did not attract the attention of the clinician, and the lack of cytological and histological examination is a mistake. This case gives us more clues that recurrent hematomas in the breast need to be looked for abnormal causes.
\end{abstract}

Case presentation: This study describes a 62-year-old woman who underwent 6 times of puncture drainage and local compression bandaging for a breast hematoma caused by blunt chest trauma. After each treatment, hematoma was restored to its original size and about $200 \mathrm{ml}$ of blood was drained each time. Hematoma resection was performed 7 months later, and the patient was diagnosed with breast cancer. Cytology and histological examination were not performed during the seven months, and patient's early diagnosis and treatment were delayed.

Conclusions: Recurring hematoma of the breast requires active search for the cause of abnormal bleeding, and tumor bleeding is a key point that cannot be ignored.

Keywords: Breast cancer; Hematoma; Breast trauma

List of abbreviations: ER: Estrogen Receptor; PR: Progesterone Receptor; Her- 2: Growth Factor Receptor 2

\section{Introduction}

Breast injuries can often be traced to blunt injuries, mostly motor vehicle accidents. Most breast injuries present with pain ecchymosis, and edema. Hematoma is a common problem after breast trauma and should be suspected in women with bruises and painful breast lumps. In the absence of shock, bleeding and other complications, it always hasn't received further surgical or imaging interventional treatment. Auxiliary bra, analgesia, compression dressing always can control the hematoma. The likelihood of developing breast cancer should be carefully assessed if signs or symptoms persist for a long time [1].

\section{Case Presentation}

A 62-year-old woman accidentally collided with her left breast on her bicycle, and an hour later, bruising and lumps were observed, and the size of the lumps did not change significantly for 2 months.
Ultrasound examination 2 months after the trauma indicated that a hypoechoic mass was observed at the left outer lower quadrant of the left breast. The doctor did not recommend further examination and treatment. About 2 months later, ultrasound examination was performed again, it indicated that no significant change in tumor size and a cystic solid mixed mass was observed in original position. Mammography presented as oval dense mass with BIRADS assessment category 4. The first breast hematoma puncture was performed on August 20, 2019 and 200ml of bloody fluid was drained out. And the compression bandage was conducted, however the tumor restored to its original size after ten days. From August to November 2019, the patient underwent 5 more puncture and drainage procedures, about $200 \mathrm{ml}$ bloody fluid was drained out after each puncture, but no cytology examination and core needle puncture was performed. Because hematoma affects the daily life of 
the patient, the patient has a strong desire for surgical treatment, and plans to be admitted to hospital for surgical treatment on November 18, 2019.

\section{Physical examination}

Bilateral breasts were basically symmetrical, a tough $5 \mathrm{~cm} * 6 \mathrm{~cm}$ mass was palpable in the outer and lower quadrant of the left breast, with good mobility, clear boundaries and with tenderness No obvious bruise or skin damage were observed on the skin. On November 22, 2019, the hematoma of the left mammary gland was resected. During the operation, the hematoma ruptured, with dark red old hematoma and brown blood clot flowing out of the lumen. The hematoma cyst wall including some normal glands was completely resected, and the cyst wall was incised and sent for pathology. Pathologist observations before the slicing indicated that part of the cyst wall is localized thickening and protrudes into the cyst cavity, with grayish white, brittle texture, rough surface and unclear boundary. Postoperative pathology showed infiltrating carcinoma of breast with cystic change. The lesion included cystic change size of $5 \mathrm{~cm}^{*} 4 \mathrm{~cm} * 4 \mathrm{~cm}$. Immunohistochemical examination indicated that: Estrogen Receptor (ER) was strongly positive, Progesterone Receptor (PR) was negative, Human Epidermal Growth Factor Receptor 2 (Her-2) was negative. The patient was diagnosed with breast cancer and underwent modified radical mastectomy of the left breast on December 11, 2019. No residual cancer was found in the breast specimens and 20 axillary lymph nodes were examined, but no cancer metastasis was found. Patients were treated with 4 cycles of pharmorubicin + cyclophosphamide chemotherapy. The patient is currently undergoing endocrine therapy.

\section{Discussion}

Breast hematomas are found in trauma, such as surgical and anticoagulant induction or abnormal blood clotting caused by blood diseases. In blunt trauma to the chest, breast hematoma is rare, with a rate of less than $2 \%$ [2]. The presence of a breast hematoma should be suspected when subcutaneous ecchymosis and painful masses are present. Hematoma can lead to necrosis of the skin, which may require surgical drainage. Severe hematomas are common in patients with clotting disorders or taking anticoagulants, and reports have shown that the use of combined anticoagulants with blunt trauma in the breasts causes huge hematomas, which require emergency surgery [2]. Most traumas are self-healing, therefore, if patients' signs or symptoms persist for more than 4-6 weeks, then their risk of breast cancer must be carefully evaluated [1].

Basic imaging examinations for breast masses included ultrasound, mammography and MRI. Mammography were postponed or omitted due to the great pain brought to patients in acute phase and ultrasound was more commonly used in clinical practice, while ultrasound showed different manifestations in different phase. In the acute stage, hematoma is manifested as hypoechoic hematoma, with acting with coagulation function, which tends to be pure hyperechogenicity [3]. In this case, the manifestations of carcinomas in the surgically resected specimens were as follows: localized thickening of the cyst wall and protrusion into the lumen with unclear boundaries; invasive carcinoma of the breast accompanied by cystic changes; while in the ultrasound examination two months after the trauma, there was also a cystic solid mass. Combined with the medical history, the radiologist considered it as the organizing manifestation of hematoma. This also suggests that a single ultrasound is flawed and mammography is complementary to ultrasound examination. When the patient has an organized hematoma that will always show mammographically as a mass of high density, a biopsy is necessary for complete evaluation.

But the clinician's inherent impression of a simple breast hematoma ignored the mammography's indication. Breast trauma would lead to changes similar to malignant breast tumors, and there was no distinguishable from malignant tumors when ultrasound and mammography were used for follow-up of breast hematomas and fat necrosis, while dynamic MRI could provide important clues [4]. The significance of enhanced CT in breast hematoma is mainly to determine the bleeding scope and the amount of bleeding. Regarding the simple traumatic non-neoplastic breast hematoma, CT examination mostly showed the hematoma in the posterior breast space. In the reported cases, the amount of bleeding was large and rapid, and arterial bleeding was considered after the final operation [2,5]. In tumor bleeding, parenchymal blood vessel hemorrhage is considered to break into the cyst cavity or to form the cyst cavity in the catheter. Due to the limited space, self-compression and hemostasis are more common. After puncture and drainage, the pressure decreases and the parenchymal vessels bleed again. There are also cases of spontaneous rupture and hemorrhage of encapsulated papillary carcinoma reported in the case report, with the increase of hematoma within a few days, huge tension causing skin necrosis and massive rupture of hemorrhage, and emergency surgical treatment is required. Therefore, the amount of bleeding of breast tumors with cystic components can also be large, which is worthy of surgeons' attention [6]. In two situations, a biopsy is necessary for complete evaluation: when there is absorption and the residual area presents a focal distortion of the parenchyma, or when there is not any absorption, in which the patient has an organized hematoma that will always show mammographically as a mass of high density.

Although a history of both cancer and trauma has been reported, there have been few studies on whether trauma causes cancer. Although wound healing and tumor growth share common physiological mechanisms, such as overexpression of cytokines and growth factors. Experimental studies have shown that the 
biochemical environment for wound healing is conducive to tumor growth. But we now think of traumatic breast cancer rupture as the cause of hematoma, not breast trauma as the cause of breast cancer considering the short course of the illness [4].

\section{Conclusion}

We should be alert to the existence of hematoma caused by tumor rupture, and cytology or histological examination is necessary. Simple puncture and drainage cannot solve repeated hematoma. If cytology and histology are negative, surgical resection is appropriate.

\section{Declarations}

\section{Competing Interests}

The authors declare that they have no conflicts of interest

\section{Consent for Publication}

Written informed consent for publication was obtained from the patient. Ethics approval and consent to participate. This study was approved by the Institutional Research Ethics Committee of the First Affiliated Hospital of Anhui Medical University. And written informed consent was obtained from the patient.

\section{Funding}

Natural Foundation of Anhui Province (No.2008085MH294)

\section{Ethical Considerations}

This research was permitted by the Ethical Committee of the First Affiliated Hospital of Anhui Medical University.

\section{Availability of Data and Materials}

The datasets used and analysis during the current study are available from the corresponding author on reasonable request.

\section{References}

1. Melissa, L, Givens (2002) Breast disorders: a review for emergency physicians. Journal of Emergency Medicine 22(1): 59-65.

2. Amin M, Jerome JP, Aguiniga, Navarrete P, Castro LC (2018) Massive Right Breast Hematoma. Clin Pract Cases Emerg Med 2(4): 357-358.

3. van Netten JP, Mogentale T, Smith MJ, Fletcher C, Coy P (1994) Physical trauma and breast cancer. Lancet 343(8903): 978-979.

4. Fink C, Lüdemann H, Wasser K, Delorme S (2002) Incidental finding of a mucinous carcinoma of the breast by dynamic MRI in a patient with a history of breast trauma (horse bite): incidental mucinous carcinoma after breast trauma. Clin Imaging 26(4): 254-257.

5. Patel K, Kimbrell BJ, Marx MV, Patrizio P, Juan AA (2009) Angiographic embolization of an expanding breast hematoma after blunt trauma: a novel approach to a rare injury. J Trauma 67(1): E14-16.

6. Yoneyama K, Nakagawa M, Hara A (2019) A case of encapsulated papillary carcinoma of the breast treated with emergency surgery due to sudden hemorrhage. Int J Surg Case Rep 65: 201-204. 